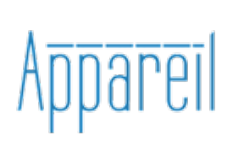

Appareil

$22 \mid 2020$

Une philosophie de l'appareil

\title{
Revenances de Jean-Louis Déotte
}

Pour Martine

Daniel Payot

\section{OpenEdition}

Journals

Édition électronique

URL : http://journals.openedition.org/appareil/3632

DOI : 10.4000/appareil.3632

ISSN : 2101-0714

Éditeur

MSH Paris Nord

Référence électronique

Daniel Payot, « Revenances de Jean-Louis Déotte », Appareil [En ligne], 22 | 2020, mis en ligne le 21

décembre 2020, consulté le 26 mars 2021. URL : http://journals.openedition.org/appareil/3632 ; DOI :

https://doi.org/10.4000/appareil.3632

Ce document a été généré automatiquement le 26 mars 2021.

\section{(2) $\odot \Theta \Theta$}

Appareil est mis à disposition selon les termes de la Licence Creative Commons Attribution - Pas d'Utilisation Commerciale - Pas de Modification 4.0 International. 


\title{
Revenances de Jean-Louis Déotte
}

\author{
Pour Martine
}

Daniel Payot

1 Walter Benjamin, à qui l'œuvre de Jean-Louis Déotte doit, entre autres, des usages prolifiques du concept d'« appareil », notait dans Sens unique : "Ad plures ire, rejoindre le nombre, signifiait mourir chez les Latins. » L'enjeu de cette formule, que le Gaffiot attribue à Plaute (un auteur comique !), était suggéré dans la phrase précédente : «Car la communauté (Gemeinschaft) de tous les morts est si gigantesque que même celui qui ne fait que parler de la mort perçoit la présence de celle-là (sie verspürt ${ }^{1}$ ). »

2 Le mot plures contient une dimension comparative ou superlative : à tout instant, les morts sont plus nombreux que les vivants; le royaume des morts est celui du plus grand nombre. C'est pourquoi toute mort individuelle possède le sens d'un ralliement : au terme de la marche, de l'avancée ou de la traversée, le singulier aura atteint le lieu d'un rassemblement majoritaire que, vivant, il ne pouvait que pressentir. Le mourir n'est pas un basculement dans le néant, il est au contraire l'événement d'une amplification. Le défunt n'est pas voué à la solitude, il entre enfin dans le destin de la foule, il trouve sa place dans la multitude, il gagne son inscription dans l'abondance.

Dans le commentaire qu'il esquissait par anticipation avant de citer l'expression latine, Walter Benjamin associait d'emblée à plures deux caractéristiques qui ne sont ni anodines, ni fortuites. Il traduisait le terme latin par « communauté » (Gemeinschaft), ce qui avait d'abord pour conséquence le glissement d'une donnée quantitative (le nombre, plus ou moins important) vers une dimension qualitative et positive : en effet, une communauté n'est pas seulement une masse, une pléthore mesurée, chiffrée, c'est, au moins, un ensemble de relations, même si on ne lui confère pas l'organicité en laquelle Ferdinand Tönnies, en 1887, discernait sa spécificité et sa différence de la société (Gesellschaft). Le règne des morts n'est pas une somme, une addition de juxtapositions et de contiguïtés, il se présente comme un tissu mouvant, une intrication de rapports, un entremêlement de séquences éloquentes.

4 La seconde caractéristique introduite par Benjamin est plus surprenante encore. Cette communauté, écrit-il, est si vaste que "même celui qui ne fait que parler de la mort » en ressent la présence, en discerne les traces. Ici encore, on passe d'une considération 
quantitative (la communauté est riesig : gigantesque, immense, énorme, géante) à une intensité qualitative: celui qui parle est affecté, dans sa sensibilité immanente, par l'effectivité de ce dont il parle. Les indices ou les « déictiques » linguistiques qu'il utilise deviennent des marques animées, des empreintes sensibles dont le locuteur, au-delà des mots qu'il emploie, éprouve en son corps le contact.

5 Ainsi Benjamin laissait-il deviner, dans la seule phrase citée, un double enseignement. Il insinuait que parler d'un mort, c'est aussi envisager le peuple dont il fait désormais partie; et il ajoutait que cette évocation n'est pas abstraite, lointaine, désincarnée, exclusivement conceptuelle, qu'elle est au contraire de l'ordre du toucher et du stigmate, parce que la profusion de ce peuple ne peut qu'affecter celui qui parle, comme si s'instaurait entre lui et la communauté des morts éveillée par ses propos une relation sensible, une réciprocité d'au-delà ou d'en deçà du discours, un entretien tangible.

6 Dans un article ${ }^{2}$ publié en 2002, Jean-Louis Déotte se demandait si - et de quelles manières - la philosophie pouvait penser la disparition, ce dernier terme entendu comme concept, mais aussi et d'abord dans le contexte d'une violence politico-militaire qui, du goulag stalinien au Nacht und Nebel hitlérien, du 17 octobre 1961 parisien aux milliers de disparus chiliens pendant la dictature d'Augusto Pinochet et aux desaparecidos de l'Argentine des années 1976-1983, a inventé « une mort pire que la mort, une mort qui n'en est pas une, un statut des corps tels qu'ils ne sont ni visibles, ni invisibles, mais avisibles ${ }^{3} »$. La philosophie, qui dans son histoire a tant médité sur la mort, peut-elle trouver les ressources d'une pensée de la disparition? Cela ne supposet-il pas qu'elle s'émancipe d'une certaine compréhension de la présence, qu'elle cesse de se soumettre, directement ou subrepticement, à ses exigences apparemment souveraines? Parvient-elle à rendre intelligible l'«avisible», ou ne peut-elle, par impuissance, que le recouvrir d'une chape d'inintelligibilité redoublant sa scandaleuse proscription, à un moment où il serait pourtant si nécessaire et si urgent d'au moins pouvoir le penser?

7 Les philosophes que Jean-Louis Déotte sollicitait alors étaient Jacques Rancière, JeanFrançois Lyotard et Jacques Derrida. Curieusement, le dernier mot était laissé à saint Augustin. Non qu'il s'agît de suggérer qu'on trouverait dans ses écrits les réponses à la question posée, mais plus vraisemblablement était-ce là l'exemple d'une pensée qui, malgré ses assurances théoriques et son indéniable inscription dans une métaphysique de la présence, ne pouvait éviter malgré tout de côtoyer une limite, audelà de laquelle s'annonçait, irrépressible, le motif d'une certaine spectralité.

En s'appuyant sur les analyses de Jean-Claude Schmitt, historien de l'Occident médiéval, et notamment sur son ouvrage Les Revenants. Les Vivants et les Morts dans la société médiévale ${ }^{4}$, Jean-Louis Déotte explicitait ainsi les trois « visions » distinguées par saint Augustin dans son commentaire de la Genèse ${ }^{5}$. Si la visio corporalis est pure perception et si la visio intellectualis se porte, "au-delà de toute image ", vers "l'indicible et l'invisible », la visio spiritualis, elle, l'intéressait tout particulièrement, en ce qu'elle mobilise l'imagination et que celle-ci, «intermédiaire et médiatrice entre sensus et mens", "reçoit et élabore des images ${ }^{6}$ ». Elle reçoit, précisément, des " "semblances de corps", également nommées species, similitudines, figurae, formae, $u m b r a e^{7} »$. Or ces mots, ainsi que deux autres termes qui leur sont immédiatement associés - persona et imago-, ont ceci de troublant qu'ils peuvent univoquement signaler des êtres matériels dont on se souvient ou dont on rêve, des êtres surnaturels 
et des morts. Persona, par exemple, est utilisé « pour désigner un être surnaturel (ange, saint, mort, voire le Christ) que le bénéficiaire de l'apparition n'a pas encore identifiés"; quant à imago, son usage ne crée "aucune différence de nature entre l'apparition de l'image d'un mort et celle de l'image onirique d'un ami qui vit au loin et dont on rêve à son insu' ${ }^{9}$.

9 Si cette univocité est potentiellement troublante, c'est en grande partie parce que, on le comprend, les fruits de l'imagination ne sont pas ici conçus comme des productions ou projections arbitraires, mais bien comme des recueils d'apparitions. La visio spiritualis est une activité dont le premier mouvement est celui d'une réception: elle est destinataire de venues, d'événements, de phénomènes qui d'abord s'adressent à elle, la hantent. C'est en cela que « la persona des morts, c'est-à-dire leur image ou semblance immatérielle » peut être source d'une impressionnante indécidabilité : certes, cette image ne possède pas l'évidence matérielle d'une perception physique, mais c'est précisément parce qu'elle se situe dans l'espace médiant entre sensus et mens qu'elle peut indifféremment être souvenir ou revenance, évocation ou habitation. C'est en cela aussi, en tant que collecte d'apparition, que le territoire de l'imago, "image spirituelle" et non "corporelle" ", peut - pas chez saint Augustin lui-même, qui " nie que les vivants et les morts puissent avoir le moindre échange direct ${ }^{10}$ ", mais dans l'imaginaire médiéval et peut-être aussi dans des imaginaires bien plus récents que ceux du Moyen Âge - s'ouvrir, au-delà des réalités physiquement attestées, à toutes sortes de visitations, d'anges et de démons, d'esprits variés et aussi de revenants et de fantômes.

10 Pourquoi était-il important pour Jean-Louis Déotte de développer de telles considérations? Certainement pas seulement parce qu'il trouvait passionnantes l'histoire des imaginaires et celle des philosophies. D'autres raisons motivaient sans doute le choix de consacrer au Moyen Âge le dernier élan d'un texte dont les premières lignes étaient : « Le temps qui vient sera celui de l'extension de la spectralité, du fait de la généralisation d'une politique héritée de la mafia consistant à faire disparaitre les corps des victimes ${ }^{11}$.»

11 S'agissait-il de suggérer une essentielle différence entre une revenance des morts historiquement et anthropologiquement liée à des rituels d'ensevelissement, c'est-àdire à une considération des corps, et une hantise spectrale irréductible à la première, parce que provoquée par l'évocation de personnes disparues soustraites à tout regard et à toute attestation? Les lecteurs étaient-il finalement invités à réfléchir, comme à l'horizon de notre temps, à des modes de visitation terriblement irréductibles à tous ceux qu'avait connus et imaginés jusqu'à nous l'histoire de l'Occident?

Jean-Louis ne nous aidait apparemment pas beaucoup à transformer ces interrogations en certitudes quand, tout de suite après avoir parlé des esprits et des revenants médiévaux, sans la moindre ligne de transition qui en préparât la teneur, il concluait abruptement son article par les deux phrases suivantes : «Il était donc inévitable que le cinéma devienne, au $\mathrm{xx}^{\mathrm{e}}$ siècle, le médium des spectres. Le cinéma, cet appareil augustinien. »

13 L'effet de montage était ici manifeste; il pourrait bien suggérer, au moins, deux orientations. En invoquant pour finir le concept d'« appareil », central et déterminant dans toute sa recherche, Jean-Louis Déotte instaurait un rapport entre ce concept et la notion de "spectralité ", comme si nous était indiquée là une hypothèse générale. L'appareil, il le définissait entre autres comme « la technique qui affecte la sensibilité », 
«qui développe un jeu autonome sur la perception et la sensibilité et, affectant une singularité, la transforme » et qui ce faisant influe sur « les connexions, les opérations, les agencements entre différentes activités humaines, espace corporel et espace d'images $^{12}$ ». L'hypothèse serait alors la suivante: cette "technique » n'est jamais quitte d'une relation singulière, complexe et à expliciter avec la mort, avec la parole qui la nomme, avec la sensibilité qui l'éprouve, avec la revenance en général.

Par ailleurs, en désignant le cinéma comme "le médium des spectres » au $\mathrm{xx}^{\mathrm{e}}$ siècle, Jean-Louis Déotte nous invitait peut-être à dégager les spécificités des connexions tissées dans la modernité entre arts, pensées philosophiques et enjeux politiques: comme s'il nous était dit que si le cinéma savait si bien capter ces spécificités, c'est parce qu'il possède une connivence singulière avec les fantômes et qu'il est en cela particulièrement capable de nous entretenir, non de la mort, dont parlent abondamment d'autres modes d'expression, mais bien de la disparition comme telle.

En écrivant, dans l'un de ses textes les plus synthétiques sur le sujet, «l'appareil, c'est ce qui prépare le phénomène à apparaitre pour "nous ${ }^{13}$ " ", Jean-Louis Déotte formulait d'une manière concise plusieurs des dimensions à ses yeux constitutives de ce concept. Il le situait d'abord, en un geste "kantien ", du côté des conditions de possibilité. Mais il y ajoutait une forme de répétition abyssale. Le phénomène, que le début de la Critique de la Raison pure définissait comme "l'objet indéterminé d'une intuition empirique ", était déjà pour Kant en lui-même un « apparaître » : «Ce qui se manifeste à nous [...] s'appelle phénomène "; « les sens nous représentent quelque chose simplement comme il apparaît $t^{14}$ [...]. » Dans la définition de Déotte, cet " apparaître » est encadré par deux autres parutions : en aval, l'expression «ce qui prépare le phénomène à apparaître " serait un simple pléonasme, s'il ne s'agissait précisément de suggérer qu'il ne suffit pas au phénomène d'être phénomène pour apparaître, proposition que Kant n'aurait que très difficilement admise, mais que nous, en revanche, comprenons bien, conscients que nous sommes (plus ou moins) de nos ignorances, aveuglements et dénégations innombrables. En amont, logiquement, si le phénomène dont il est question n'est pas spontanément ou automatiquement un apparaître, on comprend l'indispensable fonction d'une condition plus ancienne encore, d'une «préparation » qui lui ouvre le champ de sa propre manifestation.

De Kant à Déotte, on sera donc passé d'un phénomène qui, s'y l'on peut dire, n'a pas besoin d'autre chose que de sa définition pour remplir son office, à un phénomène qui n'est effectif que sous la condition de son décloisonnement, de la construction de sa propre possibilité de parution. Apparaître ne suffit pas, il faut en somme que soient au préalable réunies les conditions de possibilité de l'apparaître de l'apparaître - et c'est dans l'aire de cette ouverture, transcendantale (pour parler kantien) mais aussi d'emblée historique (pour dire avec Benjamin que tout apparaître de l'apparaître est toujours l'ouverture d'une époque singulière de la perception, de la sensibilité, de l'articulation de la loi et du corps), que le concept d'appareil (dont on notera alors la relative proximité d'assonance avec toute la chaîne sémantique de l'apparaître, apparence, apparition) trouve sa pertinence.

Cette pertinence est d'autant plus suggestive que Jean-Louis Déotte, dans toute son œuvre, aura méticuleusement déjoué tous les pièges qui menacent la pensée dès lors qu'elle semble surenchérir sur le préalable, la possibilité du possible, l'antécédence du précédent. Pas question pour lui de verser dans quelque " historialité », dans quelque fantasme de l'origine, dans quelque « jargon de l'authenticité » : l'appareil n'est pas une 
instance première, fondamentale ou fondatrice, son concept n'a rien à voir avec un transcendantal posé comme substance, structure invariante ou archétype anhistorique. $\mathrm{Au}$ contraire, son usage n'est légitime que quand les conditions de possibilité de l'apparaître de l'apparaître sont interrogées et analysées comme des inscriptions de part en part historiques, quand elles s'inscrivent dans l'histoire et y produisent les ruptures, altérations, inventions et développements que Jean-Louis aimait suggérer par le terme d'« époque » (trois de ses ouvrages en portent le titre, et peut-être n'est-il pas fortuit que l'« époque » à laquelle ils se réfèrent soit presque simultanément celle de l'appareil et celle de la disparition ${ }^{15}$ ).

Dans ce contexte, le fait que, dans la définition «l'appareil, c'est ce qui prépare le phénomène à apparaître pour "nous" ", le pronom soit placé entre guillemets mérite certainement d'être remarqué. Kant ne prenait pas de telles précautions, quand il faisait du phénomène "ce qui se manifeste à nous", sans autre indication typographique. Aujourd'hui, toutefois, le «nous» ne bénéficie manifestement pas davantage d'immédiate évidence que le phénomène. Sans doute n'a-t-il plus le statut d'un universel, d'une catégorie abstraite ; la dimension même d'une première personne du pluriel a peut-être cessé de pouvoir être évoquée d'une manière exclusivement grammaticale, formelle. "Nous» ne sommes plus seulement des opérateurs linguistiques, des «déictiques" pronominaux, et cela pourrait aussi se dire de deux façons contrastées: "nous» avons perdu l'assurance d'une présence syntaxique intangible, mais aussi: "nous» ne sommes plus pensables en termes seulement opératoires, en termes de dispositifs; « nous » revendiquons d'être reconnus dans nos singularités sensibles, dans notre irréductibilité aux catégories, aux thèmes et aux législations fonctionnelles. Et d'abord, "nous» entendons être compris comme les destinataires historiquement situés, inscrits dans leur époque, de phénomènes "préparés » à apparaître "pour "nous" »: «nous » voulons être traités comme des interlocuteurs responsables dans une époque clairement circonscrite de l'histoire de l'apparaître.

19 Il ne s'agit pas là de revendications intempestives, mais plutôt de l'un des attendus constitutifs de la notion d'appareil : pour qu'il y ait de l'apparaître, il faut qu'il y ait du "nous"; cette proposition, qui au premier abord ressemble à une lapalissade, se complexifie lorsque les guillemets autour du pronom font entendre, comme en creux, la question à laquelle nous ne savons précisément pas, ou plus, répondre: quelle première personne plurielle sommes-« nous » - autrement dit : quelle « communauté » formons-nous? Si l'époque des appareils, par hypothèse, était caractérisée entre autres par l'impossibilité de répondre à cette question, elle pourrait alors être approchée de deux manières contrastées. En un certain sens, le «nous » y est particulièrement présent, puisqu'inclus a priori dès le stade de la préparation à l'apparaître des phénomènes: "nous " sommes là d'emblée, beaucoup plus et beaucoup plus tôt que quand l'apparaitre était attribué, exclusivement ou quasi-exclusivement, à quelque intervention transcendante, magique ou divine, quand par exemple il était essentiellement le fait de la Création et de la Révélation. Pourtant, cette présence accrue du «nous», en l'occurrence, ne témoigne pas du tout d'une certitude quant à soi, d'une assurance de soi, c'est même tout le contraire: le «nous" se révèle indispensable au moment même où se retire de lui toute évidence quant à son être ou à son statut. 

demeurer possible, l'apparaître a besoin d'un « nous », mais celui-ci n'est plus concept, principe, catégorie, universalité abstraite. Qu'est-il alors, sinon un « nous » sensible, un « nous » d'affect ? Parce que la « communauté » est désormais sans contour notionnel, sans circonscription ontologique, sans définition intangible, ce qu'il reste du "nous ", c'est le corps. «Préparer » le phénomène, comme le fait l'appareil, c'est l'adresser à une réception physique, sensible, corporelle.

C'est peut-être pourquoi, à l'époque des appareils qui est aussi l'époque de la disparition, quand les corps du «nous » disparaissent, les apparitions et les revenances deviennent inéluctablement spectrales.

Telle que pensée et théorisée par Jean-Louis Déotte, l'époque des appareils pourrait bien alors prescrire une loi inconditionnelle : celle du maintien, malgré tout et à tout prix, des deux conditions de possibilité conjointes de l'apparaître "pour "nous" » que sont, aussi inévitables l'une que l'autre, l'incertitude ontologique quant au «nous » et la nécessaire attestation des corps. Il serait toujours catastrophique que l'une soit mise au service de la négation de l'autre: soit que l'incertitude quant au statut de la communauté soit traduite en termes de dévaluation ou banalisation des corps, dont l'effet serait de les livrer sans défense à quelque pouvoir arbitraire d'en disposer, soit que la réalité tangible des corps soit comprise comme un empire tyrannique ordonnant violemment la restauration d'un sens absolu de la communauté et justifiant en son nom une domination sans partage sur le «nous » réel.

Il y a là un enjeu essentiel, qui pour Jean-Louis Déotte était bien sûr d'abord politique, et qui était aussi esthétique, au sens premier du terme qui concerne la sensibilité, la faculté physique d'être affecté, la possibilité d'une réception effective des phénomènes.

Dire du cinéma, comme le faisaient les derniers mots de l'article cité, qu'il est un "appareil augustinien", c'était peut-être alors, au-delà de l'apparente boutade, suggérer que le cinéma ne peut être, comme incontestablement il l'est, l'indice de toute une époque qu'en maintenant, dans la préparation des phénomènes destinés à "apparaître pour "nous" ", une stricte et nécessaire séparation entre la visibilité des corps et la figuration de leur hypothétique " communauté ». Il s'adresse aux corps, aux sens, aux facultés réceptives, il les renvoie à l'idée d'un « nous », d'un plures susceptible d'accorder à chacun une place reconnue dans la multiplicité ; mais simultanément, il se garde de leur imposer des certitudes quant à eux-mêmes et quant à la forme de la communauté ainsi évoquée. Il ne prétend pas dire la vérité des corps et de leurs rassemblements, il sait que les phénomènes qu'il prépare à l'apparaître sont des « semblances » - « semblances de corps », à l'instar des « images spirituelles » produites par l'imagination selon saint Augustin. Si le cinéma était pour Jean-Louis Déotte un "appareil augustinien", c'était peut-être en raison du décalage qu'il manifeste inexorablement par rapport aux ontologies : montrant des corps, il expose du «nous » ce qui n'en est ni le concept, ni l'évidence abstraite, ni la figure essentielle, mais des images - des images «spirituelles » qui préparent l'apparaitre sans l'absorber dans le tout d'une figure, qui le préparent "pour "nous" ", c'est-à-dire pas pour la vérité absolue, l'universalité désincarnée ou la perfection d'un entendement infini. Pour parler de l'« apparaître pour "nous" » à l'époque de la plus grande incertitude quant au "nous », il fallait un art qui affecte nos sensibilités par le biais de la persona, terme désignant un être surnaturel mais aussi le masque de théâtre, le personnage ou l'acteur, et de l'imago, de l'apparence ou semblance des corps. 

réceptions. Il suffit pour s'en convaincre de parcourir l'anthologie publiée par Daniel Banda et José Moure sous le titre Le cinéma : naissance d'un art, qui cite des écrits de la fin du XIX ${ }^{\mathrm{e}}$ et du début du $\mathrm{XX}^{\mathrm{e}}$ siècles ${ }^{16}$. Dès les premières pages, on y découvre des propos tels que : «Lorsque ces appareils seront livrés au public, lorsque tous pourront photographier les êtres qui leur sont chers non plus dans leur forme immobile mais dans leur mouvement, dans leur action, dans leurs gestes familiers, avec la parole au bout des lèvres, la mort cessera d'être absolue. "; "Et ce merveilleux Cinématographe, qui nous rend le spectre des vivants, nous donnera-t-il, en nous permettant d'en conserver le fantôme, et les gestes, et le son de voix même, la douceur et les caresses des chers êtres disparus? ». Maxime Gorki, en 1896, parlait de " royaume des ombres », de «mouvement d'ombres, et uniquement d'ombres", de "sourires morts » et, à propos d'une séquence de jeu de cartes filmée dans un train en marche : "on dirait que ces hommes sont morts, et que leurs ombres sont condamnées à jouer aux cartes en silence pour l'éternité ». György Lukács, en 1913, disait des personnages des films qu'ils «ne sont pas des personnes » et que « les images du cinéma [...] prennent [...] un caractère irréel [...] : une vie sans présence, une vie sans destin, sans raisons, sans motifs », dont le désir « n'est que celui d'un gouffre étranger, le désir de quelque chose de lointain, dont nous sommes intérieurement retranchés». Leonid Andreev, en 1912, comparant l'image cinématographique à celle du miroir, disait d'elle : «ce n'est ni la mort ni la vie [...] mais une seconde vie, une existence énigmatique, une existence semblable à celle du fantôme ou à une hallucination ". On trouve des traces de pareilles hantises jusque chez Franz Kafka qui, vers 1920, à propos du cinéma, confiait à Gustav Janouch : "Le regard ne s'empare pas des images, ce sont elles qui s'emparent du regard. Elles inondent la conscience ${ }^{17}$. "

Jean-Louis Déotte, lui, discernait l'irruption de la spectralité dans l'image à une époque bien antérieure à celle du cinématographe et son insistance sur ce point visait aussi à interroger ce qui constituait le mode d'être spécifique des spectres au $\mathrm{xx}^{\mathrm{e}}$ siècle. Son hypothèse était que le cinéma et la photographie prolongent ce qui s'était instauré avec la peinture et les prolifiques effets de l'appareil perspectif. Cette intuition, analysée en plusieurs endroits de ses écrits, il l'avait formulée d'une manière particulièrement explicite lors de la soutenance de la thèse de doctorat d'Adolfo Vera, qui présentait un travail de recherche intitulé "L'image politique : art et disparition ", dans lequel il interrogeait le constitutif paradoxe auquel se confrontent la photographie et le cinéma quand ils s'efforcent de « représenter la disparition »-c'est-à-dire, au fond, quand ils tentent de représenter ce qui remet en cause la possibilité même de la représentation. Au milieu de l'éloge qu'il faisait de ce très beau travail ${ }^{18}$, Jean-Louis, en écho à une citation de Jacques Derrida (" "L'avenir est aux spectres” écrit Derrida pensant caractériser les technologies de télécommunication »), se demandait: " Mais l'art en Occident n'a t-il pas toujours été spectral depuis l'avènement de la perspective?» 

et l'écriture de traces de ce qui est là-bas, derrière le plan de projection ", lui suggérait l'idée que ce dispositif avait peut-être eu pour effet de faire "rentrer l'art de l'ère projective dans un monde de spectres ». Jean-Louis ajoutait, en amplifiant encore le champ de l'hypothèse : "Ne faudrait-il pas dire qu'à partir du moment où les signes visibles ont cessé de se référer au Livre (la Bible) en étant comme la face visible du lisible, alors la vérité ne s'entendait plus en termes d'incorporation, on entrait dans le monde de la représentation où dominent les signes picturaux qui certes ont bien un référent, mais sans réalité physique, donc des esprits?»

La question, dont l'examen nécessiterait de longs développements, préparait ici une autre interrogation, formulée pour saluer les résultats de la recherche d'Adolfo Vera: "pourquoi ne parle-t-on pas de l'essentielle spectralité de la peinture classique? Pourquoi cette question ne surgit-elle qu'avec les appareils dits de reproduction technique comme la photo ou le cinéma?»

La réponse, on le devine, ne pouvait-être selon lui que celle-ci : parce que l'époque qu'habitent ces appareils est aussi « l'époque de la disparition ».

Tenter d'analyser les effets sur la représentation, sur la narration, sur l'image en général et sur la notion même d'art, d'une époque qui, aux corps morts et à leurs traces, a si souvent substitué leur absolue dissolution dans l'indéterminé, le " neutre », la pure carence de toute empreinte, de toute marque, de tout ce sur quoi pourrait venir accoster le souvenir et le deuil; tenter de caractériser les façons singulières de témoigner de ceux qui, rescapés, survivants, proches, se trouvent confrontés à ce phénomène irréductible à la présence mais aussi à tout indice - qui sont hantés par cette absence qui n'offre aucune prise à ses idéalisations emphatiques, au sublime, à la transsubstantiation, à un irreprésentable transcendant: telle fut l'ambition de plusieurs textes de Jean-Louis Déotte, accompagné dans cette recherche par Alain Brossat, avec lequel il dirigea deux volumes collectifs sur ces thématiques, et par tous les contributeurs de ces ouvrages aux titres et sous-titres éloquents : L'époque de la disparition. Politique et esthétique, en 2000 ; La mort dissoute. Disparition et spectralité, en 2002. Parmi ces contributeurs, Martine Lefeuvre-Déotte, auteure de deux articles qu'on qualifiera, par la simplicité non négociable et les enjeux toujours urgents et nécessaires qu'évoque l'adjectif, de «justes » : «La politique des Mères (à propos des "folles" de la Place de Mai)» dans le premier de ces recueils, «17 octobre 1961: un crime sans transmission » dans le second.

Ensemble, ils ont essayé d'explorer les possibilités qui demeurent, malgré tout, du témoignage et de la nomination, possibilités dont ils savaient qu'elles ne sont ni naturellement, ni spontanément offertes, qu'elles doivent aujourd'hui à tout prix être construites, élaborées, préparées, si nous ne voulons pas nous résigner aux violences totalitaires et terroristes. Jean-Louis citait parfois le terrible pressentiment de Walter Benjamin, formulé en 1940 : «si l'ennemi triomphe, même les morts ne seront pas en sûreté ${ }^{19}$. » Il l'actualisait de la façon suivante : "ce sont les lieux funéraires qui fondent les topoï de l'expérience traditionnelle et donc de la narration. Si ces lieux sont indéterminables, alors, il n'y a plus d'expérience politique possible ${ }^{20}$. " Contre ce «ne plus ", il cherchait, avec Martine, avec Adolfo, avec tant d'autres en France et en Amérique latine, les conditions préservées de la possibilité de l'expérience, dans des pratiques de la parole et de l'image dans lesquelles, précisait-il, «il ne s'agit

Appareil, 22 | 2020 
évidemment pas de nommer des présences effectives, mais des présences-absences (des traces $\left.^{21}\right)$.»

Cette recherche requérait un préalable: savoir ne pas «reculer d'horreur », non pas seulement "devant la mort », comme le disait la préface de la Phénoménologie de l'esprit de Hegel, mais devant la revenance spectrale, conséquence non d'un périr laissant ouverte la voie d'une « vie qui porte la mort » et trouve les ressources d'un « maintien dans la mort même ${ }^{22} "$, mais de la disparition, qui ne laisse des corps aucun indice. Il fallait affronter cela d'abord, pour espérer ensuite deviner ce qui pourrait se substituer aux lieux et aux récits directs et reconstruire malgré tout une possibilité de nomination, d'inscription, de parole, d'articulation et d'enchânement, une manière de témoigner et de rendre hommage à ceux dont nous voulons soustraire l'«avisibilité " des corps disparus à l'incommensurable violence de leurs bourreaux.

31 À cette fin, l'œuvre de Jean-Louis Déotte constitue une très précieuse contribution et c'est elle que je voulais ici mettre en valeur, à titre d'hommage, comme l'une des plus généreuses et des plus utiles "revenances» de Jean-Louis. Dans l'article de La mort dissoute déjà abondamment cité, il écrivait: "Or des fantômes des disparus ne subsistent que de l'affect. Un affect, c'est une charge d'émotion, et dans le cas des spectres, une intensité qui cherche un destinataire.» La phrase immédiatement successive était : «La structure de l'affect est celle de l'œuvre d'art en général ${ }^{23}[. .$.$] . »$ Nous avons là, en résumé, la motivation d'un travail de recherche de plusieurs décennies qui n'aura cessé d'articuler esthétique et politique, travail éloigné de tout dogmatisme idéologique comme de tout esthétisme, soucieux sans complaisance d'établir pour chaque époque étudiée les modalités de l'affect, c'est-à-dire de l'inscription sensible, toujours historique, des corps dans les lois de leur temps.

L'œuvre de Jean-Louis Déotte ne se répartit pas en un travail sur la notion d'appareil d'un côté et en des considérations sur la disparition de l'autre, elle aborde la question de la disparition parce que les concepts d'histoire, d'expérience et de sensibilité sont lus à partir de la notion d'appareil. C'est en effet parce que son œuvre a particulièrement enrichi le concept d'appareil que ses propos sur la disparition parviennent à en établir la question d'une manière philosophiquement pertinente et politiquement nécessaire.

C'est cette hypothèse que je voulais tenter de formuler, pour tâcher de prendre la mesure des enjeux auxquels Jean-Louis ne cessait, je crois, de se confronter et dont les mots employés par Walter Benjamin dans les phrases citées ci-dessus de Sens unique permettent peut-être de suggérer l'envergure : à l'époque de la disparition, pouvonsnous encore, parlant de la mort, être affectés au point d'éprouver quelque chose comme l'empreinte d'une communauté? Mais quelle communauté de vivants formerions-nous, si nous n'étions plus capables de confier nos morts à aucun plures, à aucun "nombre ", depuis lequel leurs corps et leurs singularités éteintes continuent à faire trace " pour "nous" ", pour nos mémoires, nos pensées et nos deuils? 


\section{BIBLIOGRAPHIE}

Banda Daniel et Moure José (dir.), Le Cinéma : naissance d'un art. Premiers écrits (1895-1920), Paris, Flammarion, coll. « Champs. Arts », 2008.

Benjamin Walter, «Sur le concept d'histoire », trad. de l'allemand M. de Gandillac revue par P. Rusch, in Euvres III, Paris, Gallimard, 2000, p. 427.

Benjamin Walter, «Vestiaire de masques », Sens unique, précédé de Une enfance berlinoise, trad. de l'allemand et préface J. Lacoste, Paris, Maurice Nadeau, 1988, p. 235-237.

Brossat Alain et Déotte Jean-Louis (dir.), L'Époque de la disparition. Politique et esthétique, Paris, L'Harmattan, coll. « Esthétiques », 2000.

Déotte Jean-Louis, «La falsification par les disparus », in Alain Brossat et Jean-Louis Déotte (dir.), La Mort dissoute. Disparition et spectralité, Paris, L'Harmattan, coll. « Esthétiques », 2002, p. 215-241.

Déotte Jean-Louis, L'Époque de l'appareil perspectif (Brunelleschi, Machiavel, Descartes), Paris, L’Harmattan, coll. « Esthétiques », 2001.

Déotte Jean-Louis, L'Époque des appareils, Paris, Lignes-Léo Scheer, 2004.

Hegel Georg W. F., « Préface », La Phénoménologie de l'esprit, trad. de l'allemand J. Hyppolite, Paris, Aubier-Montaigne, coll. « Philosophie de l'esprit », 1939.

Janouch Gustav, Conversations avec Kafka, trad. de l'allemand, introduction et notes B. Lortholary, Paris, Les Lettres nouvelles/Maurice Nadeau, 1978.

Kant Emmanuel, « Analytique transcendantale », première édition, in Euvres philosophiques, tome 1 Des premiers écrits à la Critique de la Raison pure, éd. F. Alquié, Paris, Gallimard, coll. « Bibliothèque de la Pléiade », 1980, p. 979.

Kant Emmanuel, « Esthétique transcendantale », in CEuvres philosophiques, tome 1 Des premiers écrits à la Critique de la Raison pure, éd. F. Alquié, Paris, Gallimard, coll. «Bibliothèque de la Pléiade ", 1980, p. 782.

Schmitt Jean-Claude, Les Revenants. Les Vivants et les Morts dans la société médiévale, Paris, Gallimard, coll. « Bibliothèque des histoires », 1994.

\section{NOTES}

1. Walter Benjamin, «Vestiaire de masques ", Sens unique, précédé de Une enfance berlinoise, trad. de l'allemand et préface J. Lacoste, Paris, Maurice Nadeau, 1988, p. 221.

2. Jean-Louis Déotte, "La falsification par les disparus ", in Alain Brossat et Jean-Louis Déotte (dir.), La Mort dissoute. Disparition et spectralité, Paris, L'Harmattan, coll. « Esthétiques », 2002, p. 215-241.

3. Ibid., p. 215.

4. Jean-Claude Schmitt, Les Revenants. Les Vivants et les Morts dans la société médiévale, Paris, Gallimard, coll. «Bibliothèque des histoires ", 1994.

5. Jean-Louis Déotte, « La falsification par les disparus », op. cit., p. 237-240.

6. Ibid., p. 238.

7. Id.

8. Ibid., p. 240. 
9. Id.

10. Ibid., p. 237.

11. Ibid., p. 215.

12. Ces expressions se trouvent dans le texte intitulé "À propos d'Appareil ", rédigé pour présenter la revue lors de sa parution. URL: https://journals.openedition.org/appareil/86 [consulté le 29/06/2020]

13. Jean-Louis Déotte, L'Époque des appareils, Paris, Lignes-Léo Scheer, 2004, p. 102.

14. Emmanuel Kant, «Esthétique transcendantale », in CEuvres philosophiques, tome 1 Des premiers écrits à la Critique de la Raison pure, éd. F. Alquié, Paris, Gallimard, coll. «Bibliothèque de la Pléiade ", 1980, p. 782 ; « Analytique transcendantale », première édition, ibid., note $a$, p. 979.

15. Alain Brossat et Jean-Louis Déotte (dir.), L'Époque de la disparition. Politique et esthétique, Paris, L'Harmattan, coll. «Esthétiques ", 2000; Jean-Louis Déotte, L'Époque de l'appareil perspectif (Brunelleschi, Machiavel, Descartes), Paris, L'Harmattan, coll. «Esthétiques », 2001 ; JeanLouis Déotte, L'époque des appareils, op. cit.

16. Daniel Banda et José Moure (dir.), Le Cinéma : naissance d'un art. Premiers écrits (1895-1920), Paris, Flammarion, coll. «Champs. Arts», 2008. Les propos cités se trouvent respectivement aux pages $41,43,48-50,216-217$ et 226 de cet ouvrage.

17. Gustav Janouc, Conversations avec Kafka, trad. de l'allemand, introduction et notes B. Lortholary, Paris, Les Lettres Nouvelles/Maurice Nadeau, 1978, p. 213-214.

18. Les propos cités ci-dessous ont été lus par Jean-Louis Déotte lors de la soutenance de la thèse d'Adolfo Vera, le 31 mai 2012, à la Maison des Sciences de l'Homme Paris Nord. Jean-Louis avait eu la gentillesse de me communiquer leur version écrite, qui figure aussi dans le rapport de soutenance déposé auprès de l'université Paris 8.

19. Walter Benjamin, "Sur le concept d'histoire », trad. de l'allemand M. de Gandillac revue par P. Rusch, in Euvres III, Paris, Gallimard, 2000, p. 431.

20. Intervention lors de la soutenance de la thèse d'Adolfo Vera ; voir note 18 ci-dessus.

21. Id.

22. Georg W. F. Hegel, «Préface », La Phénoménologie de l'esprit, trad. de l'allemand J. Hyppolite, Paris, Aubier-Montaigne, coll. «Philosophie de l'esprit », 1939, p. 29: «Ce n'est pas cette vie qui recule d'horreur devant la mort et se préserve pure de la destruction, mais la vie qui porte la mort, et se maintient dans la mort même, qui est la vie de l'esprit. »

23. Jean-Louis Déotte, « La falsification par les disparus », op. cit., p. 229.

\section{RÉSUMÉS}

Cet article montre comment, dans la pensée de Jean-Louis Déotte, les notions centrales d'appareil (compris aussi comme condition d'apparaitre) et de disparition sont liées. C'est la disparition des corps, dans le cadre d'une stratégie d'oubli organisée par certains régimes, et aussi la structure spectrale peut-être inhérente à toute technique de représentation depuis la perspective albertienne et jusqu'au cinéma, qui sont en jeu. La spectralité permet ainsi d'envisager de manière originale les rapports entre arts, techniques et politique. 
INDEX

Mots-clés : appareil, apparaître, communauté, nous, disparition, représentation, spectralité

\section{AUTEURS}

\section{DANIEL PAYOT}

Professeur émérite, Université de Strasbourg, unité de recherche Accra (Approches

contemporaines de la création et de la réflexion artistiques), 5 allée du Général-Rouvillois, CS 50008, 67083 Strasbourg Cedex ; payot@unistra.fr 